Proceedings

\title{
Ecological connectivity efficiency: a study for Maiella National Park and Abruzzo, Lazio and Molise National Park.
}

\author{
Francesco Zullo ${ }^{1 *}$, Cristina Montaldi ${ }^{1}$, Chiara Cattani ${ }^{2}$ and Bernardino Romano ${ }^{1}$
}

University of L'Aquila, 67100 L'Aquila, Italy.

1Department of DICEAA, bernardino.romano@univaq.it (B.R.), montaldicri@gmail.com (C.M.)

2Department of MESVA, chiara.cattani@student.univaq.it

*Correspondence: francesco.zullo@univaq.it

\begin{abstract}
:
For over 30 years, the concept of the ecological network has been the subject of several strategies and projects aimed at improving ecological connectivity. The efforts to create ecological networks have been several (from the European to the regional level), but often they have not been turned into concrete actions, thus not achieving the expected results. This work focuses on a new methodological approach to create effective ecological networks through an analysis that considers both the geography of the infrastructural gaps of the main roads and the framework of the new urbanization of the involved territories. In particular, it describes a case study about the territory adjacent to two Italian parks: Maiella National Park and Abruzzo, Lazio and Molise National Park.
\end{abstract}

Keywords: ecological network; urban areas; urban planning, National Parks.

\section{Introduction}

In Italy, the ecological networks' regulatory and operative tools framework at the regional level appears to be quite wide and varied. This has also led to a general confusion both on the identification and the effectiveness of the regulatory instruments and on the real definition of the network from the territorial to the municipal level where, often, the settlement previsions could compromise its functionality [1]. This study is part of a larger project of the Abruzzo Region (Sosten\&re) which has the general aim to develop a methodology to define guidelines to support decision-making processes for the implementation of the National Strategy for the Sustainable Development (SNSvS). In this context, this work aims to help define a new approach for the creation of an effective ecological network.

\section{Area of study}

About $20 \%$ of the Italian territory is protected [2]. 25 of the 876 protected areas are rum stays neutral with regard to jurisdictional claims in published maps and institutional affiliations.

Copyright: (C) 2022 by the authors. Submitted for possible open access publication under the terms and conditions of the Creative Commons Attribution (CC BY) license (https://creativecommons.org/license s/by/4.0/).
National Parks. Several regulatory devices guarantee ecological conservation and functionality within the protected territory, while much remains to be done to safeguard connectivity outside them [3]. For this reason, the work focuses on the territory that separates the Abruzzo Lazio and Molise National Park from the Maiella National Park (Abruzzo region) which extends for over $1000 \mathrm{~km}^{2}$. These two Parks are very close, in fact, the minimum distance between them is only $8 \mathrm{~km}$. The study area covers the territory of $21 \mathrm{mu}-$ nicipalities with a total resident population of 53200 people. This area, as shown in Figure 1 , is crossed by several roads, such as SS 17 and SR 479 that run from north to south parallel to the boundaries of the Parks. The study area has a crucial role to guarantee biotic flows between the two protected areas. The knowledge of the geography and typology of the existing gaps along the roads, the spatial layout of the new transformative predictions
20

21 22 23 24 25 26 27 28 29 
as well as the preferential path of the wildlife are of strategic importance for setting up an effective ecological network.

\section{Methodology}

To analyze the regulatory framework of new urbanization, the mosaic of municipal planning tools (Planning Tool Mosaic, PTM) was created for the study area.

The PTM required the retrieval of plans at the institutional portals of the individual municipalities and at Sulmona Territorial Center Services (CST) (https://cstsulmona.regione.abruzzo.it/sit/list.html), a pre-elaboration (digitization, georeferencing, elaboration of the union framework) and the reclassification according to the homogeneous territorial zones defined by D.M. 2 April 1968, n.1444. This process involves a certain discretion in the zonal attribution; however, this is a reversible process because the original description of the area is always preserved in the database. The territorial zones are thus defined:

A) The parts of the territory concerned by urban agglomerations that have a historical, artistic character and of particular environmental value or portions of them, including surrounding areas, that can be considered to be an integral part, for those characteristics, of the agglomerations themselves;

B) The parts of the territory that have been totally or partially built up, differently from those indicated in A) zones: partially built up is intended as being those areas in which no less than $12.5 \%$ (one-eighth) of the lands surface of the area is covered by existing buildings and where the territorial density is above $1,5 \mathrm{~m}^{3} / \mathrm{m}^{2}$;

C) The parts of the territory intended for new settlement complexes, which are unbuilt or in which the pre-existing building does not reach the limits of surface area and density referred to in point B);

D) The parts of the territory intended for new settlements for industrial plants or similar;

F) The parts of the territory intended for equipment and plants of general interest.

S) The public spaces or spaces reserved for collective activities, public green or parking, with the exclusion of spaces intended for road locations.

To identify the areas under greatest risk of interference with biotic flows, road accidents involving wildlife, which occurred from 2012 to 2018 close to the main road infrastructure, were considered. These data come from the open data portal of the Abruzzo region (http://opendata.regione.abruzzo.it/). The demographic data were found at the portal of the National Institute of Statistics (Istat, https://www.istat.it/ )

\section{Results}

The current planning framework shows that six municipalities (Pacentro, Pescocostanzo, Pratola Peligna, Rocca Pia, Sulmona and Villetta Barrea) are equipped with regulatory plans prior to 1997, the year in which Italy transposed Council Directive 92/43/EEC (Habitats Directive). The plan of Roccaraso approved in 2015 is the most recent, while Rivisondoli, despite having a significant urbanized area (50 ha) and an urbanization density in line with the average value of the area $(1,61 \%)$, is devoid of planning tools. The presence of old and not updated plans, that are antecedents to the relevant regulation, shows that often these plans are not adequate to answer phenomena like the containment and reduction of environmental fragmentation and the improvement of ecological connectivity that are fundamental to avoid genetic isolation and therefore the loss of biodiversity [4].

From the PTM, it results that all the municipalities expect the expansion of D zones and in 5 of them these predictions exceeded $50 \%$ of the total. In 8 municipalities more than a quarter of the plan forecasts concern $S$ zones. In all municipal territories, the expansion of zones $C$ has been planned. In 6 municipalities more than $1 / 5$ of the total forecasts concern these areas, the maximum is recorded for Pacentro (47\%) and in a third of cases, the expected expansion value does not exceed 10\%. In 6 municipalities more than $30 \%$ of the previsions concern $\mathrm{B}$ zones, in the remaining cases for new residential areas, $\mathrm{C}$ zones were oversized.

All municipalities (except for Alfedena, Castel di Sangro, Introdacqua and Pettorano sul Gizio) are in conditions of demographic decline, losing an average of 11 inhabitants per 
year between 1991 and 2020. The municipality of Cocullo, for example, loses about 200 inhabitants in 30 years; nevertheless, it envisages an expansion of new settlement complexes (zones C) for about $18000 \mathrm{~m}^{2}, 36$ times the current value. The lack of proportionality between the provisions of the Plans and the demographic component is recognizable in $80 \%$ of cases. In other municipal areas such as Villalago and Pacentro, for example, the extension of the new settlements is about 30 times the current one. This well highlights the strong antinomy between demographic dynamics and new urban projections.

To understand the growth potential and the possible impact on environmental connection structures, the current level of implementation of each of the urban planning tools has been analyzed. The study shows that the level of implementation is very low for $\mathrm{C}$ zones. In 7 municipalities have been realized at least $30 \%$ of the previsions and only Sulmona exceeds $50 \%$, in the $2 / 5$ of municipalities instead is not reached $10 \%$. For D zones in $85 \%$ of the analyzed sample, the outdated forecasts exceed $85 \%$. Only the municipality of Scanno has almost totally realized (82\%) what was planned. A similar condition is found for zones $\mathrm{F}$ and $\mathrm{S}$, where none of the municipalities has implemented more than $50 \%$ of what was expected, except for Introdacqua which has realized about $70 \%$ of the F zones defined by the plan.

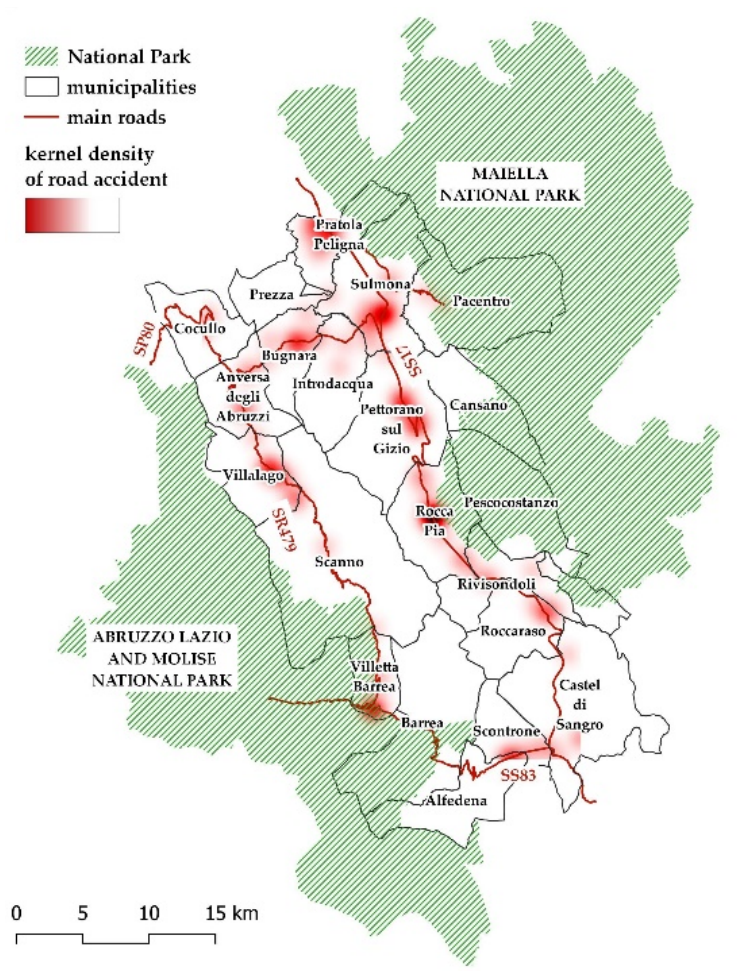

Figure 1. Framing of the study area with kernel density map of road accidents with wildlife.

To better understand the potential impact that the total implementation of the plans' projections could have on biotic flows in the area, the data on accidents with wildlife over 6 years have been analyzed. Figure 1 shows the areas where these accidents occurred most frequently. In total there were 124 collisions between motor vehicles and wildlife. The construction of new urbanized areas could further fragment this geographical area and have both direct and indirect effects on the movements of the local fauna.

Starting from these considerations, buffers of $500 \mathrm{~m}$ radius have been drawn along the sections most affected by the collisions which, as shown in Figure 1, are the SS17 and the northern section of SR 479. This type of analysis allows defining specific actions aimed at maintaining/restoring environmental continuity or mitigating possible impacts through appropriate and targeted defragmentation actions. In the buffer's areas, all the zonal typologies are identified even if with a different extension (100 ha A zones, 150 ha B zones, 
100 ha $\mathrm{C}$ zones, 205 ha D zones, 110 ha F zone, 100 ha $\mathrm{S}$ zone). In the areas where there are $\mathrm{A}$ and $\mathrm{B}$ zones, it would be appropriate to intervene through mitigation measures aimed at reducing the interactions between vehicles and fauna.

Among the possible solution, there is to separate the transit areas of vehicles from those of the fauna, through greenways or through the installation of optical, acoustic and olfactory bollards that discourage the approach of the target species to the road infrastructures. Instead, as regards the other territorial zones it is possible to intervene directly on the urban planning tools. The possible actions in these cases are several and the integrated analyses carried out, allow to satisfy more requirements jointly. These areas, for example, could be relocated or removed from the municipal plans. The first solution would be legitimate in the presence of economic and social conditions that justify the expansion of the current urban areas. In this case, the relocation would be able to satisfy both plan's requests and to reduce the potential accidents risk inevitably linked with the growth of vehicular traffic. The second solution is to freeze, reduce or eliminate certain areas. This is motivated by weak demographic energies which do not justify, for example, the planning of new settlements but also by the fact that, since many of these plans are old, these previsions have never found the economic and social pressures necessary for their realization (as mentioned above, almost all the municipalities of this area are in demographic decrease condition). This evidence, together with the fact that the degree of implementation of zones C - S is inconsistent in almost all cases, shows that the latter is an effective solution to limit the increase in environmental fragmentation and to align the predictions of urban planning tools with the real needs of the territory.

\section{Conclusion}

This work shows that in most cases, urban planning tools implemented are antecedent to environmental regulation as well as their previsions are not aligned to the current social and economic dynamics. The oversizing of the new urban areas seems to be a common feature among all plans without considering the real needs of each territory.

However, this area between the two National Parks is characterized by intense biotic flows; the lack of a strategic vision that considers the complex system of ecological networks within the new settlements could have irreversible effects on ecological functionality [5]. One of the suggested solutions is to reduce/relocate the planned urban expansion especially in those municipalities where population growth is negative.

This kind of action, resulting from accurate integrated analysis and with a systemic approach, make it possible to intervene both on the risk of collision with the wildlife and on ecological connectivity and ensure that territorial planning outlines sustainable transformation scenarios as recommended, moreover, by the Sustainable Development Goal 11 defined by the 2030 Agenda.

Funding: "This research was funded by Sost.EN.\&RE project ("Bando SNSVS 2" Ministero della transizione ecologica).

Conflicts of Interest: The authors declare no conflict of interest.

\section{References}

1. Guccione, M., et al. Le reti ecologiche nella pianificazione territoriale ordinaria. Primo censimento nazionale degli strumenti a scala locale. 2010.

2. Ispra. Available online: https://annuario.isprambiente.it/sys ind/13\#: :text=La\%20Rete $\% 20$ Natura $\% 202000 \% 20 \mathrm{in} \% 20 \mathrm{Italia} \% 20 \%$ C3\%A8\%20costituita \%2C\%20al\%20netto,a\%20mare\%20(Tabella\%203). (accessed on 20 November 2021).

3. D'Ambrogi, S.; Nazzini, L. Monitoraggio ISPRA 2012: la rete ecologica nella pianificazione territoriale. Reticula, 2013, 3,1-5.

4. Marucci, A., et al. Trasformazione degli usi del suolo, rete ecologica e rete natura 2000. Caring for our soil, $2017,73$.

5. McDonald, R. I.; Kareiva, P.; Forman, R. TT. The implications of current and future urbanization for global protected areas and biodiversity conservation. Biological conservation, 2008, 141.6: 1695-1703. 